\title{
COUGH
}

\section{Efficacy of speech pathology management for chronic cough: a randomised placebo controlled trial of treatment efficacy}

\author{
A E Vertigan, D G Theodoros, P G Gibson, A L Winkworth
}

See end of article for authors' affiliations

Correspondence to Ms A Vertigan, Speech Pathology, John Hunter Hospital, Locked Bag 1 Hunter Region Mail Centre, NSW 2310, Australia; anne.vertigan@ hnehealth.nsw.gov.au

Received 5 May 2006 Accepted 29 June 2006

Published Online First 14 July 2006
Background: Chronic cough that persists despite medical treatment may respond to speech pathology intervention, but the efficacy of such treatment has not been investigated in prospective randomised trials. The aim of this study was to determine the efficacy of a speech pathology intervention programme for chronic cough.

Methods: A single blind, randomised, placebo controlled trial was conducted in 87 patients with chronic cough that persisted despite medical treatment. Patients were randomly allocated to receive either a specifically designed speech pathology intervention or a placebo intervention. Participants in both groups attended four intervention sessions with a qualified speech pathologist.

Results: Participants in the treatment group had a significant reduction in cough (8.9 to $4.6, p<0.001)$, breathing (7.9 to $4.7, p<0.001)$, voice (7.3 to $4.6, p<0.001)$ upper airway $(8.9$ to $5.9, p<0.001)$ symptom scores and limitation $(2.3$ to $1.6, \mathrm{p}<0.001)$ ratings following intervention. There was also a significant reduction in breathing (6.8 to 5.6, $p=0.047)$, cough $(7.6$ to $6.3, p=0.014)$, and limitation $(2.3$ to $2.0, p=0.038$ ) scores in the placebo group, but the degree of improvement was significantly less than in the treatment group $(p<0.01)$. Clinical judgement of outcome indicated successful ratings in $88 \%$ of participants in the treatment group compared with $14 \%$ in the placebo group $(p<0.001)$.

Conclusion: Speech pathology is an effective management intervention for chronic cough which may be a viable alternative for patients who do not respond to medical treatment.

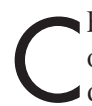
hronic cough is a common problem that has an impac on resource utilisation and quality of life. It can persist despite medical treatment based on the anatomical diagnostic protocol in $12-42 \%$ of cases. ${ }^{1-5}$ There is emerging evidence for the efficacy of behavioural approaches for the treatment for chronic cough arising from speech pathology intervention, ${ }^{6-10}$ but the role of these treatments is not universally understood in either the medical or speech pathology communities. The efficacy of speech pathology management has yet to be evaluated before it can be recognised as a viable treatment option and incorporated into protocols for the management of chronic cough.

While chronic cough is considered an entity within respiratory medicine, chronic coughing and throat clearing might be conceptualised differently in the fields of otolaryngology and speech pathology. In some voice disorders, coughing and throat clearing are considered to be phonotraumatic or vocally abusive behaviours that have contributed to, exacerbated, or perpetuated the voice disorder. These behaviours may be considered habitual and targeted in treatment programmes for voice disorders. Vocal hygiene education for hyperfunctional voice disorders includes strategies to reduce coughing and throat clearing in individuals with voice disorders and has been found to improve voice quality. ${ }^{11}{ }^{12}$ However, these treatment programmes have not been systematically applied to persons with chronic cough.

Although preliminary research into behavioural management for chronic cough indicates that this form of intervention might be a feasible treatment option, the efficacy of these treatment approaches has not been systematically investigated, making it difficult to draw firm conclusions about their potential benefits. Reports of speech pathology management for chronic cough are limited by small subject numbers, lack of comparison groups, limited standardised prospective and objective measures for voice, and the lack of prospective and randomised trials. ${ }^{8}$ Few studies of speech pathology management for chronic cough have explored treatment description and efficacy in detail.

The aim of the current study was to determine the efficacy of a speech pathology management programme for chronic cough by a prospective randomised trial of behavioural intervention. It was hypothesised that persons with chronic cough will have greater improvement in clinical outcome and symptom ratings following a speech pathology intervention than with a placebo intervention. In order to test this hypothesis, this study proposed to determine (1) whether individuals with chronic cough who received direct speech pathology intervention had a significant improvement in symptom ratings and clinical outcome; and (2) whether the extent of the change in symptom ratings was significantly different between individuals who received active treatment and those given a placebo intervention.

\section{METHODS}

A single blind, randomised, placebo controlled trial was conducted to examine the efficacy of speech pathology treatment for chronic cough. Participants were randomised to receive either Speech Pathology Evaluation and Intervention for CHronic Cough (SPEICH-C) (treatment) or an equivalent course of healthy lifestyle education (placebo). Symptom profiles were compared before and after intervention for the treatment and placebo groups along with clinical judgements of the outcome of intervention. The study was

Abbreviations: $A C E$, angiotensin converting enzyme; $F E V_{1}$, forced expiratory volume in 1 second; FVC, forced vital capacity; GER, gastrooesophageal reflux; PNDS, postnasal drip syndrome 
Table 1 Demographic data and co-morbid medical conditions of study participants that had been treated before inclusion in the study

\begin{tabular}{ll}
\hline Mean (SD) age (years) & $59.4(13.6)$ \\
Sex (M/F) & $64 / 23$ \\
Asthma* & $18(20.7 \%)$ \\
Reflux† & $41(47 \%)$ \\
ACE inhibitors & $10(11.5 \%)$ \\
Allergies & $52(59.8 \%)$ \\
PNDS & $44(50.6 \%)$ \\
Smoking & $2(2.3 \%)$ \\
Mean (SD) FEV 1 (\% predicted) & $95(20)$ \\
Mean (SD) FVC (\% predicted) & $100(21)$ \\
Mean (SD) FEV FVV $_{1} /$ FVC & $78(8)$ \\
AHRS & $8(9 \%)$ \\
\hline
\end{tabular}

ACE, angiotensin converting enzyme; PNDS, postnasal drip syndrome; $\mathrm{FEV}_{1}$, forced expiratory volume in 1 second;

FVC, forced vital capacity; AHR, airway

hyperresponsiveness.

*Previous asthma treatment included inhaled corticosteroid and long acting bronchodilator.

tPrevious reflux treatment was proton pump inhibitors. $\ddagger$ Previous PNDS treatment included topical nasal steroids and ingested antihistamines.

§Airway hyperresponsiveness to hypertonic $(4.5 \%)$ saline.

approved by the Hunter Area Research Ethics Committee and the University of Queensland Medical Research Ethics Committee. All participants provided informed and written consent for this study.

\section{Participants}

One hundred and twenty persons with chronic cough that had persisted despite medical treatment according to the anatomical diagnostic protocol referred to the speech pathology department at John Hunter Hospital, New South Wales, Australia between April 2003 and October 2004 for behavioural management of their cough were assessed for eligibility in the study. Chronic cough was defined as the presence of chronic coughing that persisted for 2 months following medical treatment based on the approach recommended by Irwin et al, ${ }^{13}$ including treatment for asthma, postnasal drip syndrome (PNDS), gastro-oesophageal reflux (GER), and withdrawal of angiotensin converting enzyme (ACE) inhibitors (if used). The severity of the cough was sufficient for participants to seek medical attention from both general practitioner and respiratory physician. Participants had undergone respiratory case history, hypertonic saline challenge, and induced sputum analysis before inclusion in the study. Significant symptoms identified during the case history were subsequently investigated and treated. Exclusion criteria included recent upper respiratory tract infection, untreated allergy, PNDS, asthma, GER, eosinophilic bronchitis, lung pathology, abnormality on the chest radiograph, chronic obstructive pulmonary disease, and neurological voice disorder. Inclusion criteria included a minimum age of 18 years and ability to travel to John Hunter Hospital.

Six of the 120 potential participants did not meet the inclusion criteria and were excluded from the study. A further 17 potential participants declined to give consent to participate in the study. Of the remaining 97 participants, 47 were randomly allocated to the treatment group and 50 to the placebo group. One participant in the treatment group and four in the placebo group did not commence their respective intervention programmes because of unexpected family responsibilities and spontaneous resolution of symptoms before treatment commenced. Three participants in the treatment group and two in the placebo group discontinued intervention through failure to contact or attend appointments. Thus, 43 participants were included in the treatment
Table 2 Examples of strategies in the treatment programme

\begin{tabular}{|c|c|}
\hline Component & Example \\
\hline Education & $\begin{array}{l}\text { No physiological benefit from cough; capacity for } \\
\text { voluntary cough control }\end{array}$ \\
\hline $\begin{array}{l}\text { Strategies to } \\
\text { reduce cough }\end{array}$ & $\begin{array}{l}\text { Identify warning signs for cough and replace with } \\
\text { modified swallow technique, pursed lip breathing } \\
\text { exercise, or relaxed throat breath }\end{array}$ \\
\hline $\begin{array}{l}\text { Reduce } \\
\text { laryngeal } \\
\text { irritation }\end{array}$ & $\begin{array}{l}\text { Increase hydration, decrease exposure to irritating } \\
\text { stimuli }\end{array}$ \\
\hline $\begin{array}{l}\text { Psycho- } \\
\text { educational } \\
\text { counselling }\end{array}$ & $\begin{array}{l}\text { Internalising locus of control; acceptance that treatment } \\
\text { is hard work; setting realistic goals }\end{array}$ \\
\hline
\end{tabular}

group and 44 in the placebo group. The mean (SD) age of participants included in the study was 59.4 (13.6) years (range 23-84); 64 were women and 23 were men. The demographic data and history of co-morbid medical conditions of the study participants are summarised in table 1 .

\section{Procedure}

Participants completed a symptom frequency and severity rating before and after the intervention. ${ }^{14}$ Twenty three different cough, respiratory, voice, and upper airway symptoms were rated on a 5-point scale from 1 (never present or absent) to 5 (present all the time or most severe discomfort ever) based on symptoms over the preceding week. Five composite scores were calculated from the symptom rating data and included a total symptom score, breathing score, cough score, voice score and upper airway score. ${ }^{14}$ The limitation of symptoms on everyday activity was also rated on a 5-point scale ranging from 1 (not limited, have done all the activities that I want to) to 5 (severely limited).

Participants were then randomised by random number generation to receive either a treatment or placebo intervention. Once the participant consented to the study, a random number between 0.000 and 0.999 was computer generated and given to the treating speech pathologist. Participants with numbers between 0.000 and 0.499 received the placebo programme while those with numbers between 0.500 and 0.999 received the treatment programme. The treating speech pathologist was not involved in the randomisation process; however, once the treatment group was allocated, the speech pathologist knew the participant's group allocation. Group allocation was concealed from participants until the postintervention symptom rating and clinical judgement of outcome had been recorded.

The intervention for both treatment and placebo groups was provided by qualified speech pathologists with experience in treating voice disorders. Participants in each group attended four individual 30 minute intervention sessions scheduled over a 2 month period. Following the postintervention rating, the treating speech pathologist made a clinical judgement of each participant's outcome as successful, unsuccessful, or partially successful. Clinical judgements were made with reference to participant's informal reports of the effectiveness of the intervention and the speech pathologist's judgement of the individual's capacity to understand and implement the strategies.

Participants allocated to the treatment programme were offered the SPEICH-C in accordance with the standard clinical procedure at John Hunter Hospital. ${ }^{9}$ The SPEICH-C comprises four components including education about the nature of chronic cough, strategies to control the cough, psycho-educational counselling, and vocal hygiene education to reduce laryngeal irritation. Examples of these strategies are 
Table 3 Comparison of mean (SD) pre-intervention symptom scores for participants in the treatment and placebo groups (Mann-Whitney $U$ test)

\begin{tabular}{llll}
\hline Score & $\begin{array}{l}\text { Treatment } \\
(\mathbf{N}=\mathbf{4 3 )}\end{array}$ & $\begin{array}{l}\text { Placebo } \\
\mathbf{( N = 4 4 )}\end{array}$ & p value \\
\hline Total symptom & $32.9(16.0)$ & $30.4(12.9)$ & 0.634 \\
Breathing & $7.5(4.1)$ & $6.9(4.1)$ & 0.591 \\
Cough & $8.6(3.0)$ & $7.7(3.4)$ & 0.119 \\
Voice & $7.0(5.8)$ & $7.8(4.7)$ & 0.892 \\
Upper airway & $8.5(6.4)$ & $7.8(5.1)$ & 0.878 \\
Limitation & $2.3(1.1)$ & $2.3(1.1)$ & 0.715 \\
\hline
\end{tabular}

outlined in table 2. These techniques were designed to improve the efficiency of voicing by reducing the load on the larynx while promoting adequate breath support and oral resonance. The education component emphasised the futility and negative side effects of repeated coughing, the benefits of cough suppression, and the capacity of individuals to develop voluntary control over cough. ${ }^{15}$ The cough suppression component required participants to anticipate when a cough was about to occur and then implement a strategy to suppress or replace the cough. ${ }^{7}{ }^{1016-19}$ The vocal hygiene component included strategies to reduce laryngeal irritation and maximise hydration in order to reduce stimulation of cough receptors. Relaxed throat breathing exercises were also provided for those participants with inspiratory dyspnoea. The psycho-educational component addressed some differences between behavioural and medical treatment and aimed to facilitate acceptance of a behavioural approach. ${ }^{9}$ This component was designed to facilitate internalisation of control over their cough and view the cough as something individuals do in response to irritating stimuli rather than a phenomenon outside of their control. This approach is commonly used to establish internalised control in other clinical populations such as stuttering and Parkinson's disease..$^{20}$ Each component in the treatment programme was addressed at least once during the course of the intervention and was revised during subsequent therapy sessions according to the needs of individual participants. The programme was tailored for each participant according to specific cough characteristics such as the pattern and degree of warning before the cough that had been identified during their case history. ${ }^{14}$ Home practice of these components was also recommended.
The placebo programme consisted of four components of healthy lifestyle education including relaxation, stress management, exercise, and diet. These components were provided during four individual sessions with the treating speech pathologist in which participants received information and home practice exercises relating to each of the components. Each component was covered at least once during the course of the placebo programme.

\section{Data analysis}

Breathing, cough, voice, upper airway, limitation, and total symptom scores before the intervention were compared between treatment and placebo groups using a MannWhitney $U$ test. The Wilcoxon signed ranks test was then used to compare scores before and after the intervention in both the treatment and placebo groups. The degree of change in symptom scores following intervention was compared between the treatment and placebo groups using a MannWhitney U test. Symptom scores were analysed by intention to treat with the pre-intervention data carried forward for post-intervention analysis. The measure of clinical judgement of treatment outcome was compared between treatment and placebo groups using the $\chi^{2}$ test.

\section{RESULTS}

There was an equivalent distribution of participants into the treatment and placebo groups according to sex and age (eight men and 35 women in the treatment group, and 15 men and 29 women in the placebo group). The mean (SD) age of participants in the treatment group was 57.5 (13.8) years compared with 61.3 (13.2) years in the placebo group. There was no significant difference in age distribution between

Table 4 Comparison of mean (SD) pre- and post-intervention symptom scores and degree of change for participants in the treatment and placebo groups

\begin{tabular}{|c|c|c|c|c|c|c|}
\hline Score & Group & Pre & Post & Difference & $95 \% \mathrm{Cl}$ & $p$ value \\
\hline \multirow[t]{3}{*}{ Total } & Treatment† & $35.4(16.0)$ & $22.7(18.0)$ & $12.7(12.7)$ & 9.0 to 16.1 & $<0.001^{*}$ \\
\hline & Placebot & $29.9(13.5)$ & $28.8(16.5)$ & $2.9(12.5)$ & -0.7 to 6.5 & 0.170 \\
\hline & Difference & & & 8.5 (13.9) & 4.7 to 14.9 & $<0.001^{*}$ \\
\hline \multirow[t]{3}{*}{ Breathing } & Treatment† & $7.9(4.1)$ & $5.0(4.2)$ & $2.9(3.6)$ & 1.8 to 3.9 & $<0.001^{*}$ \\
\hline & Placebot & $6.6(4.7)$ & $5.5(3.5)$ & $1.1(3.4)$ & 0.1 to 2.0 & $0.004^{*}$ \\
\hline & Difference $\ddagger$ & & & $2.2(3.7)$ & 0.4 to 3.2 & $<0.001^{*}$ \\
\hline \multirow[t]{3}{*}{ Cough } & Treatment† & $8.8(2.8)$ & $4.9(3.0)$ & $3.9(3.2)$ & 3.0 to 4.9 & $<0.001^{*}$ \\
\hline & Placebot & $7.5(3.6)$ & $6.3(3.5)$ & $1.2(3.4)$ & 0.3 to 2.2 & $<0.001^{*}$ \\
\hline & Difference & & & $2.8(3.6)$ & 1.3 to 4.0 & $0.003^{*}$ \\
\hline \multirow[t]{3}{*}{ Voice } & Treatment† & $7.2(6.0)$ & $4.7(5.2)$ & $2.5(4.3)$ & 1.2 to 3.7 & $<0.001^{*}$ \\
\hline & Placebot & $6.5(4.6)$ & $6.2(5.0)$ & $0.3(4.1)$ & -0.9 to 1.5 & 0.959 \\
\hline & Difference & & & $1.5(4.5)$ & 0.5 to 3.9 & $0.005^{*}$ \\
\hline \multirow[t]{3}{*}{ Upper airway } & Treatment† & $9.2(6.6)$ & $6.5(6.3)$ & $2.7(4.7)$ & 1.4 to 4.1 & $<0.001^{*}$ \\
\hline & Placebot & $7.4(4.9)$ & $7.4(5.5)$ & $0.1(4.1)$ & -1.1 to 1.2 & 0.946 \\
\hline & Difference & & & $1.5(4.8)$ & 0.9 to 4.4 & $0.002^{*}$ \\
\hline \multirow{3}{*}{ Limitation } & Treatment† & $2.3(1.2)$ & $1.6(1.0)$ & $0.7(1.1)$ & 0.4 to 1.0 & $<0.001^{*}$ \\
\hline & Placebot & $2.2(1.1)$ & $2.0(1.0)$ & $0.3(0.9)$ & 0.0 to 0.6 & $0.038^{*}$ \\
\hline & Difference & & & $0.5(1.0)$ & 0.0 to 0.8 & 0.011 * \\
\hline
\end{tabular}

†Calculated using Wilcoxon signed rank test.

‡Calculated using Mann-Whitney U test. 
Table 5 Summary of outcomes for the treatment and placebo groups

\begin{tabular}{llll}
\hline Score & Treatment & Placebo & $\begin{array}{l}\text { Treatment versus } \\
\text { placebo }\end{array}$ \\
\hline Total & & $\times$ & \\
Breathing & & $\sqrt{ }$ & \\
Cough & & $\times$ & \\
Voice & $\sqrt{ }$ & \\
Upper airway & $\sqrt{ }$ & $\times$ & \\
Limitation & $\sqrt{ }$ & $\sqrt{ }$ \\
\hline
\end{tabular}

$\checkmark$, significant improvement from pre- to post-intervention; $x$, no significant improvement from pre- to post-intervention; $\sqrt{ } \sqrt{ }$, improvemen significantly greater in treatment group than in placebo group.

groups in terms of age $(p=0.187)$, sex $(p=0.102)$, reflux $(p=0.911), \quad$ ACE inhibitor use $(p=0.526)$, allergies $(p=0.837)$, asthma $(p=0.187)$, PNDS $(p=0.914)$, or smoking $(\mathrm{p}=0.148)$.

\section{Symptom scores}

There was no significant difference in any pre-intervention symptom score between the treatment and placebo groups (table 3). The magnitude of improvement was significantly greater in the treatment group than in the placebo group for all symptom scores analysed by intention to treat (table 4). Participants in the treatment group had a significant reduction in all symptom scores after the intervention (table 4). In the placebo group there was a significant difference between pre and post-intervention breathing and cough scores but no significant improvement in total symptom, voice, or upper airway scores. Although there was a significant improvement in limitation scores after the intervention in both the treatment and placebo groups, the degree of improvement was significantly greater in the treatment group. Outcomes for treatment and placebo group are summarised in table 5 .

\section{Clinical outcome}

The clinical outcome for each participant was rated as successful, unsuccessful, or partially successful (table 6). Most of the participants in the treatment group were rated as having a successful outcome, while most in the placebo group were rated as having an unsuccessful outcome. The treatment group had a significantly higher incidence of participants with a successful outcome than the placebo group. Three participants in each group made positive progress but were considered to require additional speech pathology treatment at the conclusion of the programme to achieve satisfactory resolution of symptoms. Comparison of outcomes based on intention to treat was also statistically significant $(\mathrm{p}<0.001)$.

\section{DISCUSSION}

This study is the first randomised controlled trial of speech pathology intervention for chronic cough and is the largest investigation of speech pathology management for chronic cough reported in the literature.
The symptom data suggested that speech pathology was effective in reducing symptoms in chronic cough and that the treatment was more effective than a placebo intervention. The lack of a significant difference in pre-intervention symptom scores between the treatment and placebo groups indicated that improvements observed in the treatment group were due to the intervention rather than inherent preintervention differences between groups. Both the treatment and placebo groups showed a significant reduction in limitation scores following the respective interventions, but the degree of improvement was significantly greater in the treatment group. It might be supposed that the reduction in limitation scores was affected by both the improvement in symptoms and the positive attention provided during the treatment programme. The placebo effect could be relevant in both treatment and placebo groups; however, the degree of attention received during the intervention programme was consistent between the two groups.

The results of the clinical judgement were consistent with the symptom ratings and indicated that most of the participants in the treatment group had a successful outcome. The use of clinical judgement as an outcome measure is similar to the judgements made in everyday clinical practice and those described in previous reports. ${ }^{70}$ However, for research purposes, unblinded clinical judgements from the participant's treating speech pathologist are likely to be affected by bias and are therefore less robust than formal symptom ratings. The interpretation of the outcome of clinical judgements in this study should therefore be made with reference to the methodological shortcoming of this procedure.

Because of the single blinded design of this study and the nature of the intervention programmes, it was not possible to blind the treating speech pathologist to the type of intervention. The possibility that unconscious bias could have been conveyed to the participants during the course of intervention cannot therefore be discounted. Double blinding is not possible in studies of behavioural intervention. Despite this limitation, the participants remained blinded until after completion of the post-intervention symptom ratings.

The activities used in the placebo programme were unrelated to the cough. The lifestyle education programme was chosen for its similarity to the direct SPEICH-C whereby behaviour change was targeted over a number of sessions through education and specific activities. The placebo programme also comprised real life education rather than nonsensical or foil activities. Although the placebo programme was not specific for chronic cough, it is possible that the placebo activities such as stress management and progressive relaxation had a more direct influence on voice and cough symptoms than was previously anticipated. Comparison of treatment and placebo responses with a non-intervention control group might provide further information on the impact of the activities used in the placebo programme.

The 2 month duration of follow up chosen in the current study reflected current practice, but long term follow up as recommended by McGarvey ${ }^{22}$ was lacking in the current protocol. Long term follow up is lacking in many studies of

Table 6 Comparison of clinical judgement of outcome of the intervention between treatment and placebo groups $\left(\chi^{2}\right.$ test)

\begin{tabular}{lccc}
\hline Outcome & $\begin{array}{l}\text { Treatment } \\
(\mathbf{N}=\mathbf{4 3})\end{array}$ & $\begin{array}{c}\text { Placebo } \\
(\mathbf{N}=44)\end{array}$ & p value \\
\hline Successful & 38 & 6 & $<0.001$ \\
Unsuccessful & 2 & 35 & \\
Partially successful & 3 & 3 & \\
\hline
\end{tabular}


the medical management of chronic cough. For example, a systematic review of randomised trials of omeprazole in the treatment of chronic cough found limited follow up beyond the study period in the majority of studies. ${ }^{23}$ Further studies of speech pathology interventions in chronic cough are needed to investigate the duration of the beneficial effect.

This study provides preliminary support for the effectiveness of speech pathology management for chronic cough that persists despite medical treatment. Speech pathology intervention for chronic cough is multifactorial. This study demonstrates the effectiveness of the SPEICH-C, but further studies are needed to determine which specific components are the most beneficial. It is possible that protocols for the management of chronic cough according to the anatomical diagnostic protocol could be expanded to include treatment with speech pathology. Behavioural control of chronic cough is a management option with the potential to provide many savings in terms of healthcare resources including expensive medications and diagnostic investigations that may continue in a potentially fruitless search for an organic cause. ${ }^{24}$

Although speech pathology treatment appears to be successful in improving symptoms in persons with chronic cough, the mechanism behind the symptom improvement is yet to be determined. The education and reassurance given in the treatment programme may have resulted in a more rapid subjective improvement. Nevertheless, it is possible that processes such as muscle tension and cough reflex sensitivity could play an important role in chronic cough..$^{25-27}$ Smith et al compared cough sensitivity and ratings of the urge to cough among healthy volunteers assigned to a psychological exercise group, a cough suppression group who were advised to try not to cough, or a no intervention control group. ${ }^{28}$ The cough threshold was significantly reduced in the psychological exercise and cough suppression groups, but there was no significant difference in ratings of the urge to cough between the groups. The authors concluded that psychological factors could influence cough reflex sensitivity and that reducing concern and active suppression of the cough could raise the cough threshold. Extrapolating these results to the current study, it is possible that a speech pathology intervention directed at cough suppression could increase the threshold for cough and reduce cough sensitivity in persons with chronic cough. However, further studies of cough sensitivity are needed to confirm this proposition.

Several studies have found a beneficial effect on the larynx of adequate hydration including attenuating or delaying an increase in the phonation threshold pressure, which is the minimum amount of pressure needed to set the vocal folds into vibration and reduced risk of laryngeal injury..$^{29} 30$ Increasing hydration in the treatment group may have reduced the phonation threshold pressure and subsequent stimulation of the cough receptors.

Although the results of this study are favourable, they need to be replicated in order to achieve a higher level of evidence for the intervention, examine alternative treatment regimes, expand the range of outcome measures employed, and provide measures of long term follow up.

In conclusion, clinical judgement and symptom ratings support the hypothesis that speech pathology treatment is an effective behavioural intervention for chronic cough which could be considered a valid alternative for individuals whose cough persists despite medical intervention. Further investigations are required to understand the pathophysiological bases of the outcome of speech pathology intervention for chronic cough.

\section{Authors' affiliations}

A E Vertigan, Department of Speech Pathology, John Hunter Hospital, Newcastle, Australia
A E Vertigan, D G Theodoros, Division of Speech Pathology, University of Queensland, Brisbane, Australia

P G Gibson, Department of Respiratory and Sleep Medicine, Hunter Research Medical Institute, John Hunter Hospital, Newcastle, Australia A L Winkworth, School of Community Health, Charles Sturt University, Albury, Australia

This research was supported by a grant from Jennifer Thomas through the Hunter Medical Research Institute. PGG is an NHMRC Practitioner Fellow and AEV holds a scholarship from the NHMRC Centre for Clinical Research Excellence in Respiratory and Sleep Medicine.

Competing interests: none.

Larissa Mason and Matthew Frith provided clinical support for this research programme. Dr Susan Sherratt was involved in the development of the placebo programme.

\section{REFERENCES}

1 Kardos P. Proposals for a rationale and for rational diagnosis of cough Pneumologie 2000:54:110-5.

2 Lawler W. An office approach to the diagnosis of chronic cough. Am Fam Physician 1998;58:2015-22.

3 Ing A, Breslin A. The patient with chronic cough. Med J Aust 1997:166:491-6.

4 Marchesani F, Cecarini L, Pela R, et al. Causes of chronic persistent cough in adult patients: the results of a systematic management protocol. Monaldi Arch Chest Dis 1998;53:510-4.

5 Haque R, Usmani O, Barnes P. Chronic idiopathic cough: a discrete clinical entity? Chest 2005;127:1710-3.

6 Russell A. Non medical management of chronic persistent cough. Australian Association of Speech and Hearing Conference. Adelaide, Australia, 1991.

7 Blager FB, Gay ML, Wood RP. Voice therapy techniques adapted to treatment of habit cough: pilot study. J Commun Disord 1988;21:393-400.

8 Murry T, Tabaee A, Aviv J. Respiratory retraining of refractory cough and laryngopharyngeal reflux in patients with paradoxical vocal fold movement disorder. Laryngoscope 2004;114:1341-5.

9 Vertigan A. Speech pathology management of chronic cough. Acquir Knowl Speech Lang Hear 2001;3:62-6.

10 Gay M, Blager F, Bartsch K, et al. Psychogenic habit cough: review and case reports. J Child Psychiatry 1987;48:483-6.

11 Broaddus-Lawrence P, Treole K, McCabe R, et al. The effects of preventive vocal hygiene education on the habits and perceptual vocal characteristics of training singers. J Voice 2000;14:68-71.

12 Roy N, Gray S, Simon M, et al. An evaluation of the effects of two treatment approaches for teachers with voice disorders: a prospective randomised clinical trial. J Speech Hear Res 2001:44:286

13 Irwin R, Boulet L, Cloutier M, et al. Managing cough as a defence mechanism and as a symptom: a consensus report for the American College of Chest Physicians. Chest 1998;114:133S.

14 Vertigan A, Theodoros D, Gibson P, et al. Voice and upper airway symptoms in people with chronic cough and paradoxical vocal fold movement. $J$ Voice 2007 (in press).

15 Lee $\mathbf{P}$, Cotterill-Jones C, Eccles R. Voluntary control of cough. Pulm Pharmacol Ther 2002;15:317-20.

16 Mastrovich J, Greenberger P. Psychogenic cough in adults: a report of two cases and review of the literature. Allergy Asthma Proc 2002;23:27-33.

17 Andrianopoulos M, Gallivan G, Gallivan K. PVCM, PVCD, EPL, and irritable larynx syndrome: what are we talking about and how do we treat it? J Voice 2000;14:607-18

18 Fulcher R, Cellucci T. Case formulation and behavioural treatment of chronic cough. J Behav Ther Exp Psychiatry 1997;28:291-6.

19 Martin R, Blager F, Gay M, et al. Paradoxical vocal cord motion in presumed asthmatics. Semin Respir Med 1987;8:332-7.

20 Andrews G, Craig A. Prediction of outcome after treatment for stuttering. Br J Psychiatry 1988;153:236-40.

21 Ramig L, Bonitati C, Lemke J, et al. Voice treatment for patients with Parkinson's disease: development of an approach and preliminary efficacy data. J Med Speech Lang Pathol 1994;2:191-209.

22 McGarvey L. Idiopathic chronic cough: a real disease or a failure of diagnosis? Cough 2005;1:9

23 Chang A, Lasserson T, Kiljander T, et al. Systematic review and meta-analysis of randomised controlled trials of gastro-oesophageal reflux interventions for chronic cough associated with gastro-oesophageal reflux. BM 2006;332:1 1-7.

24 Cohlan S, Stone S. The cough and the bedsheet. Pediatrics 1984;74:11-5.

25 Choudry N, Fuller R. Sensitivity of the cough reflex in patients with chronic cough. Eur Respir J 1992;5:296-300.

26 Canning B. Anatomy and neurophysiology of the cough reflex: ACCP evidence-based clinical practice guidelines. Chest 2006;129:33-47S.

27 Ryan N, Gibson P. Cough reflex hypersensitivity and upper airway hyperresponsiveness in vocal cord dysfunction with chronic cough. Respirology 2006;11:A48.

28 Smith J, Johnson C, Brammer C, et al. The effect of a psychological intervention on the urge to cough. American Thoracic Society 2005;A40:A113.

29 Casper J, Murry T. Voice therapy methods in dysphonia. Otolaryngol Clin North Am 2000;33:983-1002.

30 Solomon N, DiMattia M. Effects of a vocally fatiguing task and systemic hydration on phonation threshold pressure. J Voice 2000;14:341-62. 